(2) Open Access Full Text Article

\title{
A protocol for topographic-guided corneal repair utilizing the US Food and Drug Administration- approved Wavelight Contoura
}

This article was published in the following Dove Press journal:

Clinical Ophthalmology

23 March 2017

Number of times this article has been viewed

\author{
Manoj Motwani \\ Motwani LASIK Institute, \\ San Diego, CA, USA
}

Correspondence: Manoj Motwani

Motwani LASIK Institute,

4520 Executive Drive, Suite

230, San Diego, CA 92121, USA

$\mathrm{Tel}+\mathrm{I} 8585540008$

Emaildrmmlj@gmail.com
Purpose: To demonstrate how Wavelight Contoura can be used to repair corneas damaged by trauma and prior poor surgical outcomes.

Methods: Four representative eyes are presented that show different scenarios in which highly irregular corneas can be corrected with Wavelight Contoura using a protocol (named the San Diego Protocol) designed to use the information in Contoura processing. Both laser-assisted in situ keratomileusis (LASIK) and photorefractive keratectomy (PRK) were used.

Results: Highly aberrant corneas with large amounts of warpage can be corrected safely with the Wavelight Contoura system. The San Diego Protocol requires individual analysis of each case with decisions based on the level of warpage and the level of epithelial hyperplastic compensation. The need for a second refractive power equalization procedure should be planned for.

Conclusion: Contoura measured refraction can be integrally used as part of the San Diego Protocol to safely repair highly warped corneas. The refractive outcomes show dramatic improvement in vision, best-corrected visual acuity (BCVA), refraction, and topographic uniformity.

Keywords: corneal ablation, laser-assisted in situ keratomileusis, photorefractive keratectomy, refractive surgery, corneal irregularity, corneal trauma, decentered excimer laser ablations, radial keratotomy, higher order aberrations

\section{Introduction}

The repair of highly irregular corneas using topographic-guided ablation has been available for some years outside of the US, and development of the Wavelight system led to the US Food and Drug Administration (FDA) approval for primary eyes. ${ }^{1-3}$ In the FDA approval, the correction of prior distortions and corneal warpage (not to be confused with corneal warpage syndrome from contact lens wear; corneal warpage is used in the labeling to denote extreme corneal irregularity) is listed as a precaution, and not a contraindication, and hence procedures repairing past surgery, trauma, or corneal disease are not expressly off label with Wavelight topographic-guided ablation but are actually under the FDA labeling only with a precaution. ${ }^{4}$

The repair of corneal issues is an area that is fraught with lack of full understanding even outside the US and has been virtually non-existent in the US until 2016. Furthermore, there has also been a lack of full understanding thus far as to the use of the measured astigmatism and axis from the Wavelight topography-guided software. The market name has been Contoura, but the repair function is often referred to as topography-guided custom ablation treatment (T-CAT). Much of the current literature 
with T-CAT internationally has dealt with the treatment of keratoconus combined with corneal cross-linking. ${ }^{5,6}$ There has been less information concerning other forms of corneal reconstructive repair such as corneal trauma, past laser ablation decentration, and radial keratotomy (RK) irregularity correction and precious little in the way of a protocol that can be used to make decisions on how to proceed with such cases.

This study was undertaken to show four examples of corneal repairs stemming from both surgical and traumatic causes. Although each repair must be undertaken as a case unto itself and must be evaluated individually, we have developed a protocol that allows for an understanding of how to make decisions concerning corneal repair. It is this protocol that has been named the San Diego Protocol.

The fundamental problem with repairs is the extent of irregularity of the cornea and how much it affects the pre-operative (pre-op) correction. The decisions on this must be made on a case-by-case basis by understanding where the visual axis lies in the distortion. Treatment with topographic-guided ablation can be affected by corneal epithelial compensation, as epithelial hyperplasia will change the thickness of the epithelium to thin over "high" spots and thicken in "low" spots. Although instruments such as optical coherence tomography (OCT) and the Artemis very high frequency (VHF) scanner can show the extent of epithelial compensation, many clinics (including ours) do not have these devices, thus making it difficult to tell how much epithelial compensation is affecting the Contoura measured astigmatism, and even the spherical power. Even if epithelial hyperplasia information is available, this information cannot be integrated into Contoura to change the treatment, and it is only useful for diagnostic purposes.

The use of Contoura measured astigmatism in repairs derives directly from previous work done by the author demonstrating the superiority of using Contoura measured correction in primary eyes to create a uniform cornea (Motwani and Lemonis, unpublished data, 2017). To make matters more complicated, the presence of several corneal irregularity may significantly effect the ability to properly measure the manifest refraction. All of these factors must be taken into consideration when undertaking a corneal repair with topographic-guided ablation.

\section{Methods}

All repair procedures were performed by one surgeon at one center performing corrections with the Wavelight Contoura/T-CAT (topography-guided customized ablation treatment) system. All patients provided written consent to have their data published in this paper.

If a flap was created, the Moria M2 microkeratome with microspecialties blades was used. If a pre-existing flap had been created, it was re-lifted and used again. For photorefractive keratectomy (PRK) cases, epithelial debridement was performed using diluted alcohol placed in a $9.5 \mathrm{~mm}$ Reichert well, with the epithelium removed by dry Wek-Cel sponge and/or epithelial scraper.

All scans of the cornea were done with the Topolyzer Vario and processed using Contoura WaveNet, and procedures were performed on the Wavelight EX500 laser. ${ }^{7}$ Higher order aberration (HOA) ablation maps as shown in the examples were obtained by zeroing out the sphere and cylinder on the Contoura/T-CAT treatment-planning page. Multiple images were obtained, at least three usable, consistent images per eye were the minimum required for treatment. The smallest ablation zone the FDA-approved software can treat is $6 \mathrm{~mm}$, making taking scans on highly irregular corneas challenging as in our experience getting enough information for a $6 \mathrm{~mm}$ treatment can be more challenging than for a $5 \mathrm{~mm}$ treatment, which is the preferred ablation zone for many surgeons outside of the US.

As part of the surgical planning, the effect of the ablation on the spherical power must be ascertained using Zernike polynomials. On many patients, this procedure is different in the US than in the rest of the world as we do not have hyperopic Contoura. C4 and C12 denote the defocus and higher order spherical aberration, respectively, and must be equalized. ${ }^{6}$ This equalization process is used to determine the spherical effect of tissue removal by the aberration repair to calculate the effect on the final power of the cornea. $\mathrm{C} 4$ and $\mathrm{C} 12$ comparisons were done by inputting the measured astigmatism and axis (or no astigmatism at all if a highly aberrant corneal normalization is being attempted) and then comparing the $\mathrm{C} 4$ and $\mathrm{C} 12$ values in the Zernike tab on the treatment-planning page. Equalizing the two values allows you to estimate how much spherical change will occur, and the spherical power must be adjusted accordingly. Unfortunately, because Contoura has not been approved for hyperopic corrections, those aberration ablations that shifted the sphere into hyperopic territory cannot be ascertained by simply placing a hyperopic number into the sphere box, as the Zernicke values will not be computed as it is an unapproved indication. Instead, the change in value must be ascertained by changing the myopic direction and looking for the change in $\mathrm{C} 4$ and $\mathrm{C} 12$ to equalize them. For example, if the $\mathrm{C} 4$ is 1.2 and $\mathrm{C} 12$ is 0.1 , then there must be a 1.1 change to 
equalize the two. If the sphere is already plano, then myopic correction must be added to increase the $\mathrm{C} 4$ the same amount of difference. For example, in this case, the difference is 1.1, so enough myopia must be added to make the $\mathrm{C} 42.3$, and the absolute value of that will result in the amount of hyperopia the ablation will correct. This is a cumbersome system but necessary because of the limited approval obtained. If this is not done, there will be surgical surprises with the power of the cornea significantly off target. It is possible to automate this function in the software, and I believe Wavelight should update the Contoura/T-CAT software to show the amount of sphere necessary to equalize the $\mathrm{C} 4$ and $\mathrm{C} 12$. This protocol differs from past protocols in two specific ways: first, it has to incorporate changes for the US as hyperopic Contoura is not FDA approved, and second, it incorporates what the authors have learned with Contoura on primary eyes to incorporate Contoura measured astigmatism and axis into the repair protocol. Due to these changes, I have given this repair protocol its own name - the San Diego Protocol.

\section{The San Diego Protocol}

1. Perform a manifest refraction under cyclopleged conditions and also in meiotic non-cyclopleged conditions. It is important to compare these two to determine the effect on peri-pupillary coma. I have found that usage of the meiotic manifest refraction is often more useful in repairs as it minimizes the effect of peri-pupillary coma on the manifest refraction.

2. Ascertain the level of irregularity and attempt to discern where the visual axis is on the topographic map. If the cornea is severely irregular, and the patient is looking through a flatter area, it is likely the patient will be hyperopic and some of the hyperopic correction will be eliminated with the corneal repair. If the patient is looking through coma created by a warpage, then the astigmatism may change during measurement in mydriatic vs meiotic conditions. At this point, a decision must be made whether to attempt a full refractive correction or to only perform the HOA repair to normalize the cornea and perform a refractive correction after 3-6 months as a planned second procedure. As a rule, our experience has demonstrated that refractive correction and aberration repair together works best for displaced laser ablations, traumatic scar repair, and RK irregularity that is not severe.

3. Obtain multiple Topolyzer Vario images and perform careful comparisons to obtain similar, consistent images. Although our tolerance for mean astigmatic axis deviation (MAD) in primary Contoura planning is 1 degree, we do allow up to 2 degrees of variability when performing a repair.

4. Zero out the sphere and cylinder on the Contoura surgical planning page and examine the type of aberration on the ablation map. Enter the measured astigmatism and axis if desired and then compare the $\mathrm{C} 4$ and $\mathrm{C} 12$ and equalize the sphere.

5. Obtain a Scheimflug device thickness map such as a Pentacam map and also obtain an ultrasound pachymetry as Scheimflug devices can have greater variability and error in cases of prior refractive surgery. Ensure that sufficient tissue is available to perform the laser correction.

6. By pressing the right mouse button when the arrow is on the ablation map on the Contoura treatment-planning page, the ablation depth can be determined at various areas. The amount of tissue removal can change by decreasing the diameter of ablation. At times, due to limited information on the Topolyzer due to the extent of aberration, treatment at a $6 \mathrm{~mm}$ zone may be required.

7. Before performing the correction, ensure you explain to the patient that although you are attempting to correct as closely as possible, the repair reconstructive process may take a second procedure to finish the full correction.

8. Decide after 3-6 months on the type of second procedure. If high levels of epithelial compensation existed and significant aberration still exists, then a repeat topographicguided procedure is recommended. If little aberration exists, then treatment with wavefront optimized (WFO) laser profile is also possible.

\section{Methods continued}

\section{Case I - Severe RK-induced corneal irregularity}

This case involved massive surgical warpage from prior RK. A 72-year-old surgeon who had RK to treat hyperopia attempted 30 years prior, with further RK revision attempted 15 years ago. A laser-assisted in situ keratomileusis (LASIK) flap was made with a Moria M2 microkeratome (Figure 1).

The patient's left eye was treated by creating a flap with the Moria M2 microkeratome in the highly irregular cornea. Increased ocular pressure under suction creates a more normalized shape in an RK eye and allowed for a flap to be made. The use of the microkeratome also is less stressful on the cornea as no dissection is needed, and the RK slits usually do not separate.

The initial procedure was purely HOA removal, the repair function to normalize the cornea. The second procedure corrected the Contoura measured astigmatism and the spherical power. 

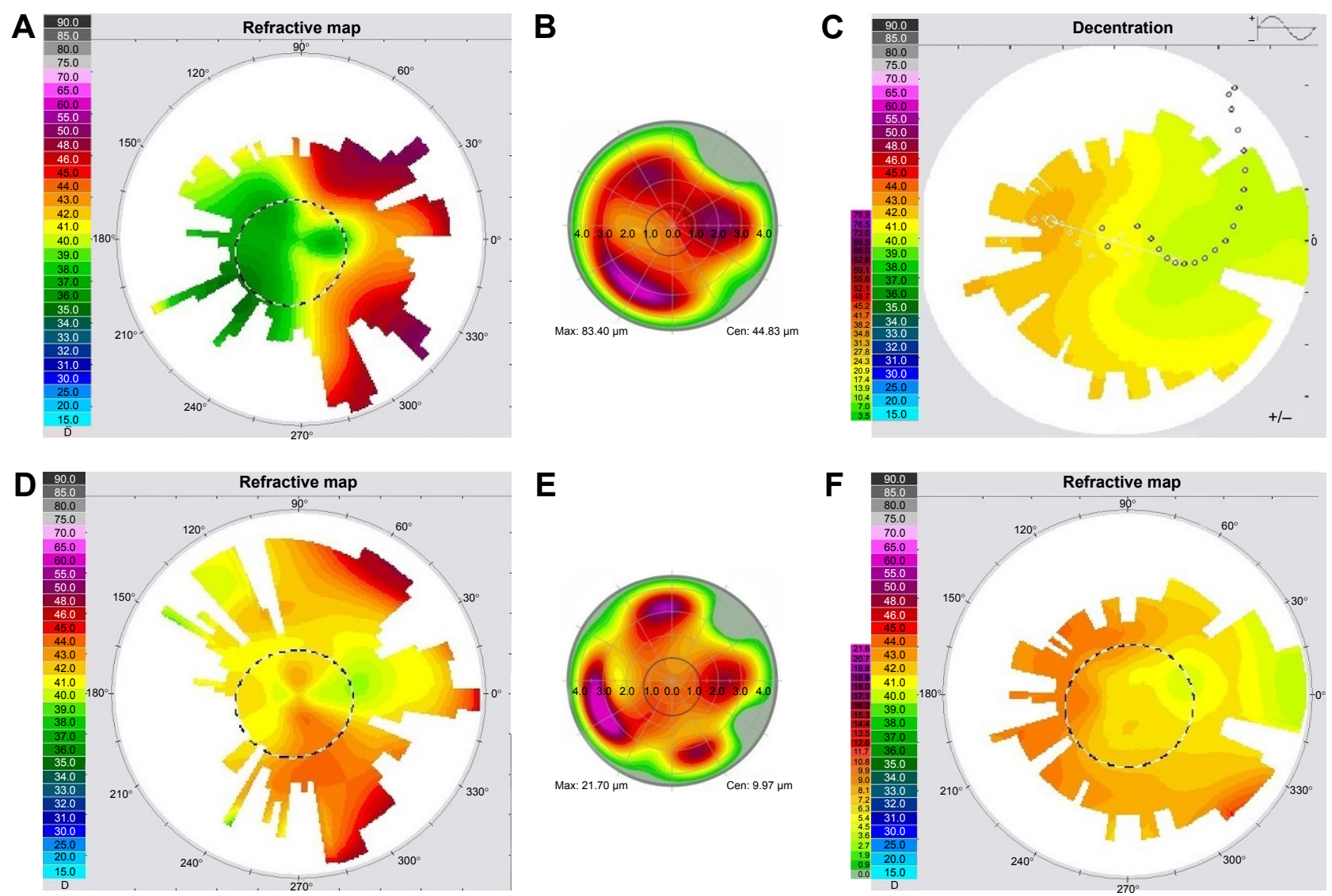

Figure I Case I. (A) Pre-op; (B) HOA ablation pattern first procedure; (C) pre-op Fourier decentration map; (D) post-op initial correction at 3.5 months; (E) second procedure HOA ablation pattern; (F) post-op 2 months after second procedure.

Abbreviations: HOA, higher order aberration; post-op, post-operative; pre-op, pre-operative.

First treatment: $0 \mathrm{D}$ sphere, 0 D cylinder (pure repair, no refractive error treated at all).

Second treatment: refractive correction including Contoura measured astigmatism manifest $\mathrm{Rx}(3.5$ months postoperative [post-op] initial correction): $+1.25,-0.75 \times 53$.

Measured Contoura treatment: plano, $2.59 \times 17$; WFO, +0.75 (hyperopic Contoura not available in the US).

C4/12 compensation: +1.3 D.

Compensation for astigmatism increases from $-0.75 \mathrm{D}$ (manifest) to $-2.59 \mathrm{D}$ (measured) $=+0.92 \mathrm{D}$.

Final spherical calculation: $+2.22-+1.25=+0.97$. A clinical decision was made to treat +0.75 with $W F O$ and leave patient at a resultant $+0.22 \mathrm{D}$.

\section{Case 2 - Repair of central corneal distortion from penetrating trauma}

A 42-year-old male had a full thickness central penetrating corneal trauma via a pair of scissors at age 3 . The procedure was done via PRK to attempt to decrease some of the scarring associated with the trauma. The patient had 1-2+ haze/ central corneal scarring (Figure 2). A $9.5 \mathrm{~mm}$ Reichert well was used with diluted alcohol applied for 30 seconds to remove the epithelium. Procedure was performed with the Wavelight EX500 using Contoura HOA removal and then WFO treatment for hyperopia.

Pre-op manifest refraction: $+3.25,-0.25 \times 10$ with BCVA of 20/40 (Figure 2A).

Measured Contoura treatment performed as PRK: plano, $-0.25 \times 54$ followed by WFO of +1.00 (partial treatment of the hyperopia as clinical uncertainty of epithelial hyperplasia affecting spherical component due to long-standing trauma) (Figure 2B).

$\mathrm{C} 4 / \mathrm{C} 12$ compensation $=$ equal, no compensation necessary.

Second treatment: performed via LASIK 3 months after initial procedure using the EX500 and Moria M2 microkeratome: $+2.25 \mathrm{D}$ as WFO with $6.5 \mathrm{~mm}$ ablation zone.

\section{Case 3: Severe decentration of excimer laser ablation}

A 63-year-old female had LASIK performed 9 years prior on a Visx laser (unknown if with tracking) with severe decentration of the ablation in the right eye (Figure 3). The original flap was re-lifted, and ablation was performed using the 

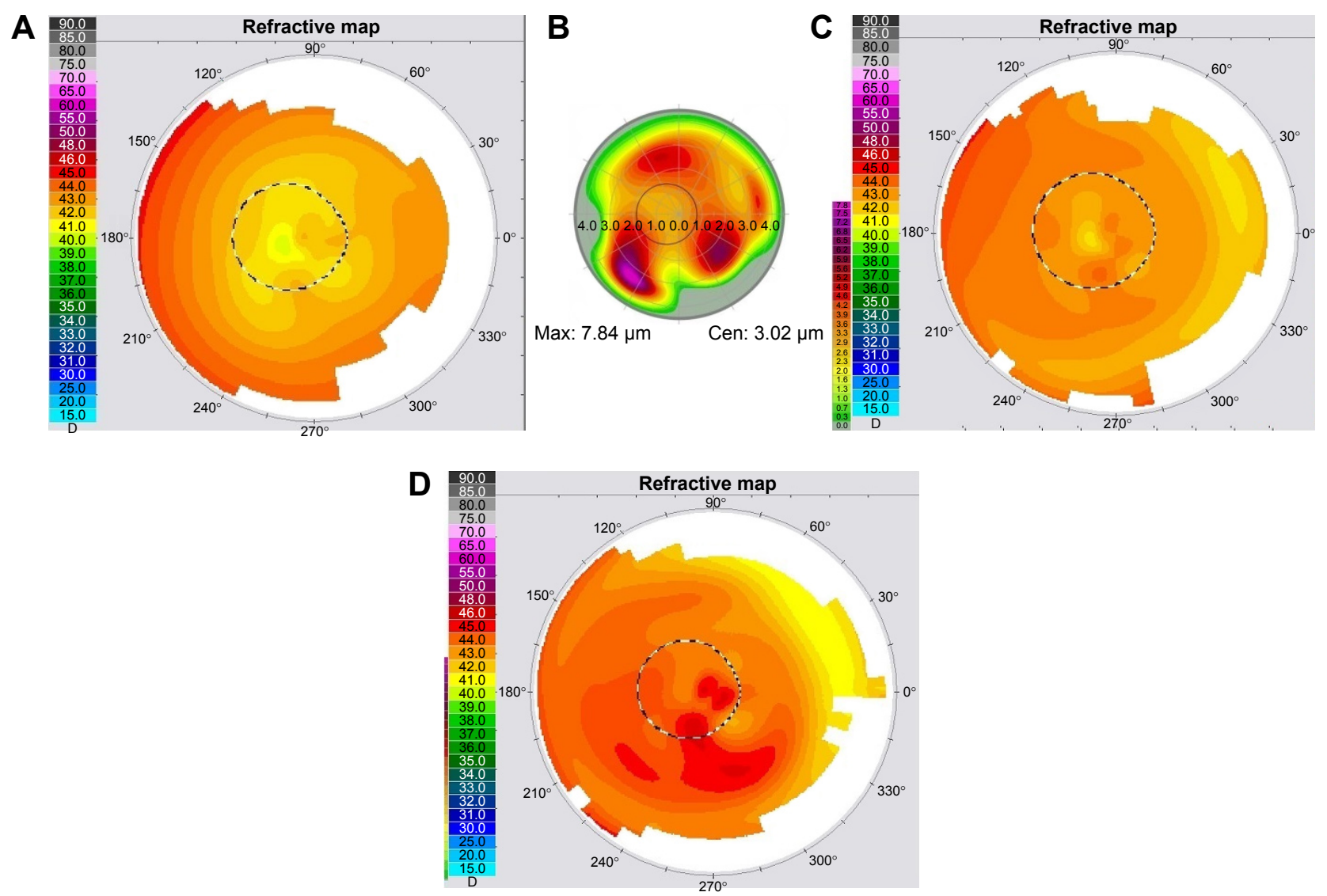

Figure 2 Case 2. (A) Pre-op; (B) HOA ablation pattern for first procedure; (C) post-op 3 months; (D) post-op 3 months after second procedure (WFO). Abbreviations: HOA, higher order aberration; post-op, post-operative; pre-op, pre-operative; WFO, wavefront optimized.

Wavelight EX500 with Contoura and then WFO treatment to correct the hyperopia.

Pre-op manifest Rx: $+2.00,-1.00 \times 175$.

Pre-op Contoura measured correction: plano, $-1.23 \times 161$ followed by $\mathrm{WFO}+1.50$.

$\mathrm{C} 4$ and $\mathrm{C} 12$ compensation: +0.50 .

Astigmatism compensation from manifest to measured $=+0.015$.
Final sphere calculation: $+2.00-0.65=1.35$ rounded up to +1.50 for WFO treatment.

\section{Case 4: Elliptical ablation from eye tilt during} excimer laser ablation

A 24-year-old patient had a high myopic WFO correction on the Wavelight EX500 and tilted her eye during the original correction. The patient ended up with an elliptical ablation
A

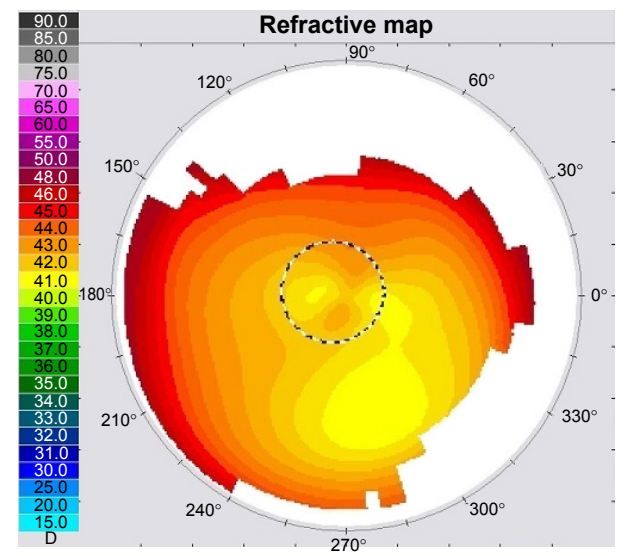

B

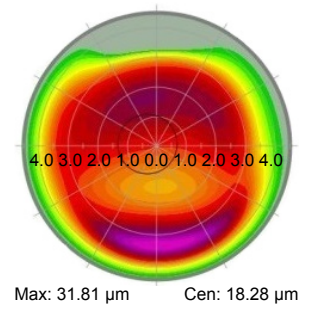

Max: $31.81 \mu \mathrm{m} \quad$ Cen: $18.28 \mu \mathrm{m}$

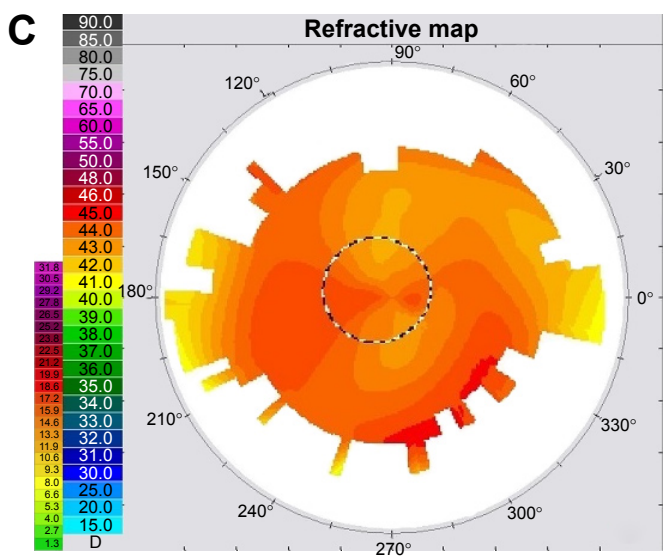

Figure 3 Case 3. (A) Pre-op; (B) HOA ablation pattern; (C) post-op 5 months.

Abbreviations: HOA, higher order aberration; post-op, post-operative; pre-op, pre-operative. 
resulting in a visual axis close to the periphery of the ablation. The patient had completely different refractions in dark and light room settings, as the coma induced by the decentered ablation would increase the manifest astigmatism. It was noted in this case that the manifest astigmatism amount was decreased in a room with bright lights and was similar to the measured Contoura amount (Figure 4). The procedure was performed as PRK, as the primary procedure was performed as Epi-Lasik. A $9.5 \mathrm{~mm}$ Reichert well was used with diluted alcohol applied for 30 seconds to remove the epithelium. Ablation was performed with the Wavelight EX500 with Contoura.

Pre-op manifest refraction: mydriatic conditions: $+1.00,-1.50 \times 90$.

Meiotic conditions: plano, $-0.75 \times 90$.

Contoura measured refraction: $-0.44 \times 14$.

Contoura performed correction: plano, $-0.25 \times 14$ (Figure 4A).

C4/C12 compensation: -0.15 (minimal so left untreated).

\section{Results}

\section{Case I: Severe RK-induced corneal irregularity}

Pre-op manifest Rx: $+6.75,-3.75 \times 60$ with BCVA of 20/30.

Pre-op Contoura measured astigmatism: $-2.54 \times 4$ (Figure 1A).

The HOA ablation pattern for first procedure (repair) shows a massive amount of inferonasal steeping (90 $\mu \mathrm{m})$ with flattening superior and superolaterally (Figure 1B).

The Fourier decentration map shows large amount of coma (Figure 1C).

Post-op 1 day first procedure: 20/25 with refraction of $+1.50 ;$ BCVA $=20 / 20$ (repair performed only, no refractive correction).
Post-op 3 months first procedure: vision of 20/30, $\mathrm{Rx}=+1.25,-0.75 \times 53$. BCVA $=20 / 20$ (Figure 1D).

The HOA ablation pattern for second procedure shows quadrafoil (Figure 1E).

Post-op enhancement 2 months: plano and 20/20 (Figure 1F).

\section{Case 2: Repair of central corneal distortion from penetrating trauma}

Pre-op Rx: $+3.25,-0.25 \times 10$ (Figure 2A); BCVA =20/4020/50 (Figure 2A).

The HOA ablation pattern shows what appears to be trefoil modified by the abnormal traumatic scar pattern (Figure 2B).

Post-op 3 months: +2.25 with BCVA of 20/25 (Figure 2C).

Second procedure: WFO treatment of +2.25 at $6.5 \mathrm{~mm}$ zone.

Post-op 3 months second procedure: +0.50 and 20/30; BCVA 20/30 (Figure 2D).

\section{Case 3: Severe decentration of excimer laser ablation}

Pre-op manifest Rx: $+2.00,-1.00 \times 175$; $\mathrm{BCVA}=20 / 20$ (Figure 3A).

Pre-op Contoura measured correction: plano, $-1.23 \times 161$ followed by WFO +1.50.

HOA ablation pattern shows correction of decentration by flattening of area superior to ablation and steepening of area inferior to ablation (Figure 3B).

Post-op 5 months: 20/20 and refraction of plano (Figure 3C).
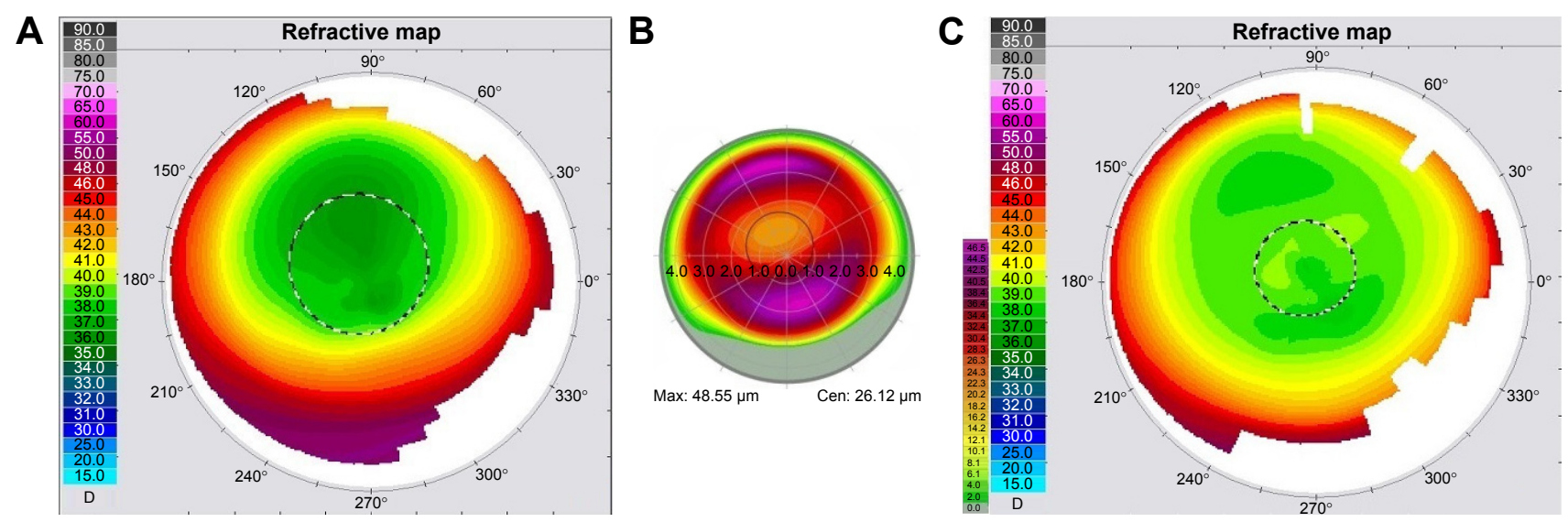

Figure 4 Case 4. (A) Pre-op; (B) HOA ablation pattern; (C) post-op 9 months.

Abbreviations: HOA, higher order aberration; post-op, post-operative; pre-op, pre-operative. 


\section{Case 4: Elliptical ablation from eye tilt during excimer laser ablation}

Pre-op manifest refraction: dark conditions: $+1.00,-1.50 \times 90$.

Lit conditions: plano, $-0.75 \times 90$.

Figure 4A shows the elliptical off-center original ablation.

Figure 4B shows the HOA ablation pattern, which flattens inferiorly and steepens superiorly.

Contoura measured astigmatism: $-0.44 \times 14$.

Contoura correction performed: plano, $-0.25 \times 14$.

9 months post-op: plano and 20/15 (Figure 4C).

\section{Discussion}

Case 1 is an example of a very severe corneal warpage/ irregularity resulting in a very large amount of coma. It was noted that the patient's visual axis appeared to be through a keyhole area that was an extension of the flat part of the cornea, and it was theorized that repair would likely eliminate much of his hyperopia due to his dramatic irregularity. Surgical planning was performed with SL of Wavelight, and it was decided at that time to simply perform the repair without any astigmatism correction. It should be noted that the Contoura measured astigmatism was not dramatically different from that at the second procedure 3.5 months later. In retrospect, the astigmatism could have been corrected at the initial repair, but due to the severe irregularity, according to the San Diego Protocol, only HOA repair was initially corrected. LASIK was the procedure of choice as the amount of ablation was so large $(89.43 \mu \mathrm{m}$ of tissue removal at the deepest point) that haze would have been a serious problem with PRK. A $9.5 \mathrm{~mm}$ flap was made without issue using the Moria M2 microkeratome, as increasing the pressure in the eye "inflated" the cornea decreasing the distortion from the RK allowing a flap to be made. Using the microkeratome makes a smooth flap without "bridges" needing no dissection that could tear the RK slits. The flap was re-lifted without issue with careful dissection to perform the second procedure. The outcome was dramatic only after treating the aberrations and no refractive error in the first procedure - the patient noticed a large difference in vision the next day and had achieved 20/25. The patient is now $20 / 20$ and plano with continued re-compensation of his epithelium 1 month post-op second procedure.

Case 2 is an example of corneal distortion from scarring secondary to penetrating trauma. Removal of his distortion was able to bring him to a BCVA unattainable pre-Contoura and normalization of his cornea, that of 20/30+2 at 2-month post-op PRK. It is yet unknown how much of his decrease of BCVA is from traumatic scarring, amblyopia due to the trauma occurring at a young age, or due to epithelial recompensation still underway. What is clinically evident is that the patient has a clarity of vision with $\mathrm{a}+1.50$ correction not experienced in his lifetime and the extent of uniformity of his topographic map even at 1-month post-op. C4 and C12 comparison was equal, and a clinical decision was made to under-treat the hyperopic correction initially due to the type of distortion that was being treated as there was concern that removing the irregularity might affect the spherical correction. This concern turned out to be unfounded, as the spherical correction remained similar and the second procedure essentially corrected the residual hyperopia. In retrospect, this could have been done as one procedure. The second procedure was performed as a LASIK by patient request, as he did not wish to have the downtime. This was deemed to be safe by the amount of tissue removed during the PRK, and clinical examination indicated that the residual scar was too deep to be further treated by surface tissue removal. His resultant vision of 20/30 is limited at this point by residual scar and perhaps some amblyopia. The patient does notice that his visual field is now much wider, his cortical processing continues to change over time to increase usage of this eye, and he can see objects, motion, and people from the side of this eye where before he could see very little.

Case 3 is an excellent example of a marked decentration of laser ablation, something that was surprising as it occurred less than a decade ago during a time of laser trackers, and attention being paid to centration of laser corrections. (As an aside, we have already seen and repaired other significant decentrations of laser ablations using devices with current, but slower trackers. It may well be that certain trackers are simply not as reliable as surgeons think.) A decision was made to fully treat the refractive correction. Her correction changed little from dark to light room conditions. Contoura measured astigmatism and axis was used, $\mathrm{C} 4$ and C12 equalization showed a correction of $\sim+0.50$, so a WFO of +1.50 was performed on top of the T-CAT correction. This patient was initially myopic, but at 1 week refracted to plano and remained that way at 1 month and later examinations. At 5 months, she was stable at 20/20 and is now happy with her vision. In this case, we were able to perform the topographic-guided HOA repair and correct the refraction in one procedure.

Case 4 is an example of an elliptical ablation caused by a tilted eye. I believe this problem has gone unrecognized and is caused by a nervous patient not fixating on the aiming light in their central vision but in their paracentral vision. This causes 
a tilt, usually inferiorly, but the tilt is not enough to prevent the tracker from locking on. This patient's original correction was with a Wavelight EX500 using a WFO procedure. The laser never had issues with fixation or tracking. The original correction was a surface ablation (Epi-LASIK), so PRK was used for Contoura correction. This patient had dramatically different refractions in a dark vs light room, showing the effect of the coma caused by the elliptical, decentered ablation. The ablation map shows a significant removal of tissue inferiorly to even out the ablation, something that was achieved with excellent results. In this particular case, the topographic-guided HOA repair was performed at the same time, resulting in good vision and corrected refraction in one procedure.

The main reasons for refractive surprises after repairs are based on the epithelium. If the epithelium has re-compensated in a way to blunt a deviation or aberration, the outcome will not be as intended as full measurement of the aberration cannot be done. ${ }^{8}$ As we know from past experience, residual aberration can combine with lower order astigmatism to create an entirely new refraction, and a second topographicguided procedure will be likely once the epithelium has become uniform again. Although in the past, 3 months was thought to be sufficient for epithelial stability to occur, there is some clinical and scientific evidence to show that waiting longer may be necessary in some patients. Some international physicians firmly advocate waiting 6 months for epithelial uniformity before conducting a second procedure. There is some evidence from examining aberration reduction polynomials in our primary Contoura studies using the Layer Yolked Reduction of Astigmatism protocol to support waiting 6 months, although we have undertaken second corrections at 3 months when shown to be stable (Motwani and Lemonis, unpublished data, 2017).

Essentially, the epithelium functions as a masking agent because of hyperplasia. ${ }^{9}$ It will blunt aberrations and smooth over small ablation areas making them unable to be fully mapped by the Topolyzer. We have no existing FDA-approved technology to combine a measurement of epithelial hyperplasia with a topographic-guided ablation, so in cases of significant epithelial compensation, a "refractive surprise" may occur leading to the need for a second topographic-guided procedure. Therefore, careful patient selection needs to be conducted, and informed consent detailing the possible need for a second procedure must be obtained. The authors expect this protocol to be a reliable corneal reconstructive tool, but in our practice, we counsel each patient to expect a second procedure due to the difficulty in accurately calculating the final overall power of the cornea. This is not exclusive to Contoura/T-CAT. Our pre-topography-guided experience with repairing old small ablation zone hyperopic corrections with the much larger, more regression-resistant Wavelight hyperopic ablation pattern has resulted in small hyperopic ablations being "pushed up" through the epithelium resulting in unexpectedly myopic post-operative results.

\section{Conclusion}

The use of topographic-guided ablation in the rest of the world has essentially revolutionized the treatment of corneal warpage and aberrations. ${ }^{3}$ Topographic-guided reconstruction of the cornea is an extremely powerful tool, but one that requires care and proper patient preparation and consent.

\section{Acknowledgments}

The author acknowledges Sissimos Lemonis of Wavelight GmbH for concept and theory and Ron Pei, OD, for content and editing.

\section{Disclosure}

Dr Motwani has received a grant from Alcon for a previous unrelated study. The author reports no conflicts of interest in this work.

\section{References}

1. Byrne J. Topography-guided ablation: a promising option for patients with irregular corneas. Prim Care Optom News. 2012;14(2):13.

2. United States Food and Drug Administration. WaveLight ALLEGRETTO WAVETM Excimer Laser System - P030008. Available from: http://www. accessdata.fda.gov/scripts/cdrh/cfdocs/cfpma/pma.cfm?id=p030008. Accessed November 15, 2016.

3. Kanellopoulos AJ. Topography-modified refraction (TMR): adjustment of treated cylinder amount and axis to the topography versus standard clinical refraction in myopic topography-guided LASIK. Clin Ophthalmol. 2016;10:2213-2221.

4. WaveLight ${ }^{\circledR}$ Excimer Laser Systems [Product Information]. Fort Worth, TX: Alcon Laboratories, Inc; 2016.

5. Krueger RR, Kanellopoulos AJ. Stability of simultaneous topographyguided photorefractive keratectomy and riboflavin/UVA cross-linking for progressive keratoconus: case reports. J Refract Surg. 2010;26(10): S827-S832.

6. Shetty R, D'Souza S, Srivastava S, Ashwini R. Topography-guided custom ablation treatment for treatment of keratoconus. Indian J Ophthalmol. 2013;61(8):445-450.

7. Allegretto Wave Eye-Q. Scanning Spot LASIK Laser System. Addendum Procedure Manual T-CAT. Topography-Guided Treatments. Erlangen, Germany: WaveLight GmbH.

8. Kanellopoulos AJ, Aslanides IM, Asimellis G. Correlation between epithelial thickness in normal corneas, untreated ectatic corneas, and ectatic corneas previously treated with CXL; is overall epithelial thickness a very early ectasia prognostic factor? Clin Ophthalmol. 2012;6: 789-800.

9. Sumit G, McColgin AZ, Steinert RF. Phototherapeutic Keratectomy. San Francisco, CA: American Academy of Ophthalmology; 2013. 
Clinical Ophthalmology

\section{Publish your work in this journal}

Clinical Ophthalmology is an international, peer-reviewed journal covering all subspecialties within ophthalmology. Key topics include: Optometry; Visual science; Pharmacology and drug therapy in eye diseases; Basic Sciences; Primary and Secondary eye care; Patien Safety and Quality of Care Improvements. This journal is indexed on

PubMed Central and CAS, and is the official journal of The Society of Clinical Ophthalmology (SCO). The manuscript management system is completely online and includes a very quick and fair peer-review system, which is all easy to use. Visit http://www.dovepress.com/ testimonials.php to read real quotes from published authors. 